\title{
Aktueller Entwicklungsstand und Perspektiven der Schulterendoprothetik
}

B chergleichszahlen, so herrscht hierzulande immer noch eine große Zurückhaltung gegenüber der Schulterprothetik. Die USA, Frankreich und die Schweiz liegen weit vor uns. So manchem Patienten wird vor seiner geplanten Schulterprothesen-Implantation von halbinformierter Seite geraten, die Operation aufzuschieben oder gar abzusagen. Die schlechte Presse erklärt sich zwangsläufig aus der bei uns sehr niedrigen Implantationsfrequenz. Mangelnde Operationspraxis muß zu Mißerfolgen führen, und diese ziehen weit größere Kreise als die vielen, aber anonym bleibenden, guten Erfolge beim Schultergelenksersatz.

Große charismatische Persönlichkeiten wie Charles Neer in USA, Didier Patte in Frankreich und Norbert Gschwend in der Schweiz haben in ihren Ländern viel für den Durchbruch der Schulterprothetik geleistet. Mit ihren Erfolgen setzten sie neue Impulse und stimulierten die weitere Verbreitung der Schulterimplantate. Auch in Deutschland gibt es mit Engelbrecht, Kölbel und Zippel Autoren, die aufgrund ihrer Prothesen-Entwicklungen weltweit zitiert werden. Noch vor Neer implantierten Engelbrecht sowohl als auch Zippel zementierte Schulter-Totalprothesen. Die ersten Stress-Analysen an der Scapula und Finite Element- Untersuchungen wurden weltweit erstmals anfangs der
80 er Jahre in Berlin unter Friedeboldt erarbeitet. In den letzten Jahren hat im deutschsprachigen Raum ein Boom an Neuentwicklungen stattgefunden. So haben Fleega, Gerber, Hertel, Habermeyer, Resch, Rüther und Thabe eigene Prothesen auf den Markt gebracht. Von dieser Seite her ist der Anschluß an den internationalen Standard erreicht.

Wo liegen nun noch bestehende Hindernisse, welche Versäumnisse gilt es aufzuarbeiten? An erster Stelle müssen hier die Kostensituation und die Budgetierung für die Krankenhäuser genannt werden. Im Gegensatz zur Knie- und Hüftprothetik gibt es für den totalen Schultergelenksersatz keine Fallpauschale! Lediglich für die Hemi-Endoprothese besteht ein Sonderentgelt, das dem Anschaffungspreis der Kopfprothese entspricht, aber keinesfalls die damit für den Träger verbundenen Kosten nur in etwa abdeckt. Dies führt letztlich zur Verwendung von Billig-Implantaten. Der zu Kostenbewußtsein verpflichtete Operateur wird geradezu gezwungen, auch wenn es notwendig wäre, aufden Einbau einer Totalprothese zu verzichten, da andernfalls sogar die Sonderentgeltregelung entfiele. Berücksichtigt man noch, daß bei den Verhandlungen mit den Krankenkassen den Operationsabteilungen Fallzahlbegrenzungen auferlegt werden, so wundert man sich nicht mehr, wenn einer orthopädischen Universitätsklinik im Jahr 25 Schulterprothesen „zugestanden"werden. Will man also die Situation verbessern, müssen zugunsten der Patientenversorgung eine längst fällige Fallpauschale für die Implantation der Schulter-Totalprothese und eine Abänderung der Quoten-Zuteilung zwischen Hüft- und Knie-Implantation und Schultergelenksprothetik gefordert werden.

Schultergelenksprothetik mußzukünftig mit Professionalität vorangetrieben werden. Nach F. A. Matsen erfordert der prothetische Eingriff am Schultergelenk eine erheblich größere Anforderung an den Operateur als vergleichsweise am Knie- und Hüftgelenk. Die lange learning curve bei geringer Fallzahl macht es im Sinne der Patientenversorgung notwendig, die Patienten an spezialisierte Einrichtungen zu überweisen. Die dort mit Zuverlässigkeit erzielbaren Resultate werden in Zukunft dazu beitragen, daß die Schulterprothetik den Stand erreicht, der heute im Hüft- und Kniebereich vorgelegt ist.

Ziel des folgenden Beitrages ist es, den aktuellen Entwicklungsstand und die Perspektiven der Schulter-Endoprothetik aufzuzeigen.

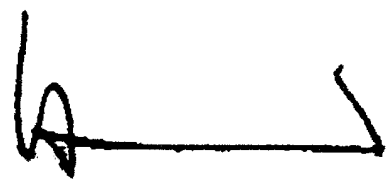

P. Habermeyer 